\title{
Quantum nanoscience
}

\author{
Nanoscale systems are ideally suited to study quantum mechanical effects and explore these as resources for \\ emerging quantum technology such as quantum sensing, communication or computing.
}

( o we need to focus on quantum in nanotechnology? Is quantum mechanics not integral part of all nanoscience?

Yes, it is. When it comes to the length scale of atoms and molecules, quantum mechanics is always at play. And yet, it is often not at the heart of the research. In many areas of nanoscience and nanotechnology, quantum mechanical effects can either be ignored to a good extent, require some minor consideration, or are fundamental to understand the system. But they are not the properties and characteristics that make the system of interest to research. On the contrary, there are fields in nanoscience that explicitly employ nanoscale systems to explore quantum mechanical effects - for example, coherence, state occupation or entanglement - and use quantum mechanics as a resource for emerging technology applications, such as quantum computation and communication, or quantum sensing. To this end, different degrees of freedom can be used such as the charge, the electron or nuclear spin of atoms, molecules, or semiconducting quantum dots, as well as photons or vibrations. The progress seen in nanoscience over the past few decades now enables exquisite control over the nanoscale structures through fabrication and synthesis approaches. Researchers can now tailor quantum mechanical effects at will with high finesse.

Initiated by Andreas Heinrich and Andrea Morello, for this Focus issue, a group of researchers from different fields of nanoscience and nanotechnology review the striking developments in the areas where nano and quantum science meet. They outline the underlying physical principles of the research field - a field they coin quantum coherent nanoscience - and look into the future, describe important roadblocks encountered in the subfields and the technological prospects.
One of these areas showing tremendous progress over the past decade is quantum photonics. In their Review, Ravitej Uppu et al. use the example of quantum-dot devices to present the governing physical principles of deterministic photon-emitter interfaces and the different photonic building blocks. The authors showcase how a modular photonics approach can lead to scalable quantum hardware, borrowing attractiveness from the mature integrated photonics foundry technology, which, according to the authors, will enable scale-up.

Closely related is the Comment by Chao-Yang Lu and Jian-Wei Pan. The authors take a look at the future quantum internet and identify components where nanotechnology in the form of quantum-dot single-photon sources can provide viable solutions to enable long-distance quantum networks. They argue that semiconductor quantum dots are well suited to enable quantum key distribution, long-distance quantum teleportation, entanglement distribution or quantum computation.

In a second Comment Maud Vinet targets a different area at the intersection of fundamental and applied research, namely silicon spin qubits. She analyses why, despite the striking characteristics of individual silicon qubits, this technology is not yet at par with more mature quantum computing platforms such as superconducting, trapped ion or cold atom qubits. Then, the author outlines that joined efforts of the quantum computing and electrical engineering communities are needed to unlock the full benefits of the platform, which, on the long term, shall bring competitiveness of silicon spin qubits for large-scale quantum computers.

Finally, our Focus on quantum nanoscience also features primary research: Jiaojian Shi, Weiwei Sun et al. present an all-optical methodology to suppress fluorescence blinking in core-shell quantum dots. The method, based on ultrafast mid-infrared laser pulses that manipulate the exited dark state of the quantum dot, may find application in real-time single-particle tracking or super-resolution microscopy techniques, but may also translate to other single emitters such as vacancy centres in diamond or defects in $2 \mathrm{D}$ transition metal dichalcogenides.

This research Article is accompanied by a News \& Views from Anna Rodina, who not only summarizes the physical mechanism of the blinking control, but also collects important questions that follow from this study and anticipates how similar methodologies based on mid-infrared pulses may extend to neighbouring fields. It is also Anna Rodina who points out that back in 1996 Moungi Bawendi, the lead author of this study, was among the researchers that reported fluorescence blinking in individual nanocrystals first (Nirmal, M. et al. Nature 383, 802-804; 1996) - what an illustration of perseverance and dedication to (nano)science.

With this Focus issue we highlight an area in nanoscience that gained traction over the past decade, both in fundamental research and for quantum technology applications. The area brings together chemists, physicists and material scientists with engineers and computer scientists and is, as much of nanoscience is, truly interdisciplinary. We are confident that we are just witnessing the beginning of quantum nanoscience and that science and technology explicitly based on quantum effects will play an important role in successfully tackling the societal challenges of the 21st century. We, the editors of Nature Nanotechnology, would be delighted to accompany this journey and promote the beautiful science that emerges along the way.

Published online: 9 December 2021 https://doi.org/10.1038/s41565-021-01058-0 